\title{
Septischer Schock und systemisches U. Müller-Werdan Entzündungsreaktions-Syndrom - Der konkrete Fall
}

\author{
Septic shock and systemic inflammatory response syndrome - \\ case report
}

\section{Anamnese}

Bei einem 47-jährigen Patienten mit einer Fettleberhepatitis und Alkoholkrankheit kam es zu einem Atemstillstand mit Aspiration. Eine Bronchiallavage erbrachte in drei verschiedenen Proben jedes Mal den Nachweis von zahlreichen Pseudomonas putida-Keimen. Darüber hinaus fanden sich Enterobacter cloacae, vereinzelt Staphylococcus aureus und sehr wenige hämolysierende Streptokokken der Gruppe C. Trotz testgerechter antibiotischer Behandlung entwickelte sich eine Aspirationspneumonie mit konsekutiver Sepsis und Multiorganversagen (ARDS; katecholaminpflichtiger hyperzirkulatorischer Schock; akutes Nierenversagen). Monitoring und Behandlung des Patienten erfolgten auf der Intensivstation.

\section{Diagnostische Maßnahmen}

Wie so häufig, ließ sich auch bei diesem Patienten ein Keimnachweis nur initial in der Bronchiallavage-Flüssigkeit, nicht jedoch im Blut erbringen. Das klinische Bild mit den typischen hämodynamischen Befunden (siehe unten) begründete die Diagnose eines septischen Schocks. Die Diagnose wurde erhärtet durch die Bestimmung des Sepsis-Scores nach Elebute und Stoner (siehe Kapitel Diagnostik Tab. 2), der weit oberhalb des „Cut-offs“ von 12 Punkten gemessen wurde.

\section{Verlauf der hämodynamischen Parameter im septischen Schock}

Prominentes Merkmal der Herz-Kreislauf-Situation bei Sepsis ist die ausgeprägte periphere Vasodilatation, die auch bei hyperzirkulatorischer Kreislaufsituation zum septischen Schock führen kann. Die hämodynamische Situation des 47-jährigen

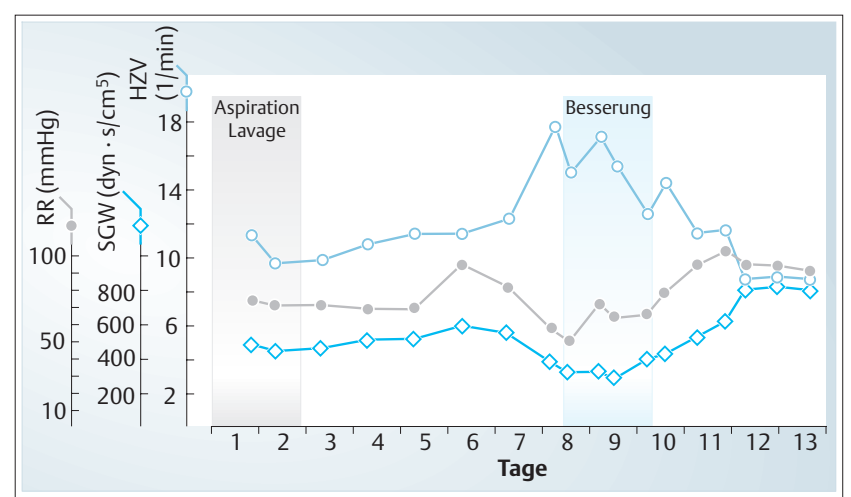

Abb. 1 Herz-Kreislauf-Situation eines Patienten mit einer Pseudomonas-Sepsis. (SGW: systemischer Gefäßwiderstand; HZV: Herzzeitvolumen; RR: mittlerer arterieller Blutdruck).

Patienten mit der Pseudomonas-Sepsis ist hierfür typisch (Abb.1). Der Verlauf des systemischen Gefäßwiderstands als Ausdruck der Nachlast zeigt den Schweregrad der septischen Vasodilatation. Nach einer initialen Befundstabilisierung kam es ab dem 7. Tag zu einer Verschlechterung mit Abfall der Nachlast und des Blutdrucks. Parallel dazu sank die Herzleistung nicht etwa $a b$, sondern das Herzzeitvolumen nahm im Gegenteil kompensatorisch zu auf weit übernormale Werte. Erst mit der anhaltenden klinischen Besserung ab dem 9. Tag normalisierten sich der systemische Gefäßwiderstand, der Blutdruck und die Herzleistung sanken wieder ab. Parallel dazu besserte sich das klinische Bild eindrucksvoll: Das Fieber fiel und die ausgeprägten Ödeme verschwanden, das ARDS ging zurück (erkennbar an den Beatmungsparametern), ebenso wie das Nierenversagen und der septische Schock besserte sich mit Rückgang des Katecholaminbedarfs. Die klinische Besserung drückte sich auch in einem Abfall des Sepsis-Scores nach Elebute und Stoner aus. Universität Halle-Wittenberg · Ernst-Grube-Straße 40 • 06097 Halle · Tel.: 0345/5572816, -2601 Fax: 0345/5572072 · E-Mail: ursula.mueller-werdan@medizin.uni-halle.de 